\title{
Fabrication and bioresorbability of Ag- and Ta-containing amorphous calcium phosphate films formed on titanium substrates by RF magnetron sputtering
}

\author{
Jun Wu ${ }^{\mathrm{a},}{ }^{*}$, Kyosuke Ueda ${ }^{\mathrm{a}}$, Takayuki Narushima ${ }^{\mathrm{a}}$ \\ ${ }^{a}$ Department of Materials Processing, Tohoku University, 6-6-02 Aza Aoba, Aramaki, Aoba-ku, Sendai, Miyagi 980-8579, Japan \\ *wu.jun.s8@dc.tohoku.ac.jp
}

\begin{abstract}
$\underline{\text { Abstract }}$
Silver (Ag)- and tantalum (Ta)-containing amorphous calcium phosphate (ACP) coating films were fabricated on titanium substrates by radiofrequency magnetron sputtering for biomedical applications. The sputtering targets were hot-pressed into $10 \mathrm{~mol} \% \mathrm{Ag}$-containing B-tricalcium phosphate sintered compacts with and without 8 mol\% Ta. The fabricated ACP coating films were dense and smooth, with all elements distributed homogeneously along the depth direction. In addition, Ag existed as ions in the ACP regardless of the presence of Ta. The resorbability of the Ag-containing ACP coating films in simulated body fluids was suppressed by the addition of Ta. The concentration of detected $\mathrm{Ag}$ ions was higher in diluted nutrient broth solution than in Tris- $\mathrm{HCl}$ solution.
\end{abstract}

\section{Introduction}

Because titanium (Ti) can directly connect to living bones, i.e. osseointegration, as observed at the optical microscopic level, it has been widely used in the orthopedic and dental fields for long-term implantation [1,2]. However, the strength of fixation between Ti implants and bones is influenced by the state of the bone and the possible dimension of the implant [3,4]. To obtain rapid and strong fixation of implants, we focused on amorphous calcium phosphate (ACP) coating films fabricated by radiofrequency (RF) magnetron sputtering on Ti implants [5-9], which have been reported to enhance the bone-forming ability because of its high bioresorbability [10,11]. ACP is known as the precursor of apatite and dissolves rapidly in vivo [8,12], indicating that ACP coating films first improve bone formation during the early stage of implantation and then completely dissolve to achieve a direct connection between the bone and Ti implant; this is defined as osseointegration.

Adding other elements to calcium phosphate glass can control its bioresorbability. For example, the addition of tantalum ( $\mathrm{Ta}$ ) (0-0.5 mol\%) to the $\mathrm{SiO}{ }_{2}-\mathrm{ZnO}-\mathrm{SrO}-\mathrm{CaO}-$ $\mathrm{P}_{2} \mathrm{O}_{5}$ glass system has been reported to suppress its dissolution in de-ionized water, because Ta can act as a glass-network-former [13]. However, to our knowledge, the addition of Ta to ACP coating film has not been investigated.

The long-term survival of implants in the human body greatly depends on both bone compatibility and absence of bacterial infections. Surgical site infection caused by bacteria adhering to the surface of the implants affects the long-term performance of implants and often leads to their removal [14,15]. To overcome these problems, Ag, [16] which has antibacterial activity even at very low concentrations, has been considered for use on the surface of implants. ACP coating films applied to the surface of implants with well-controlled bioresorbability, as is the case with the addition of Ta, would serve as an effective carrier of Ag and would control the Ag release; thus, Ta and $\mathrm{Ag}$ added ACP coating films may improve bone compatibility and achieve antibacterial activity concurrently.

In order to evaluate the in vitro resorbability of Ag-containing ACP coating films with and without Ta, immersion tests were conducted in simulated body fluids (SBFs). In our previous study, nutrient broth (NB) solution was adopted as an SBF that can be used for the antibacterial test [17]. However, the concentration of Cl ions in the NB solution is much lower than in the human body. Therefore, in order to simulate the biological environment of human body, the concentration of $\mathrm{Cl}$ ions in the solution should be increased to the same level as in the human body. For this reason, Tris(hydroxymethyl)aminomethane-buffered solution adjusted with hydrochloric acid (Tris-HCl) was used as a more biologically relevant SBF.

The aim of this study was to fabricate Ag- and Ta- containing ACP coating films on Ti substrates using RF magnetron sputtering. The composition, phase, morphology, and structure of the films were evaluated. The in vitro resorbability of the ACP coating films was also evaluated by immersion tests in SBFs. The dissolution behavior of the coating films with and without Ta was investigated.

\section{Material and experiments}

Mixtures of $\beta$-tricalcium phosphate ( $\beta$-TCP, Taihei Chemical Industrial Co., Ltd., Osaka, Japan), silver oxide ( $\mathrm{Ag}_{2} \mathrm{O}$, Wako Pure Chemical Industries, Ltd., Osaka, Japan), and tantalum pentoxide ( $\mathrm{Ta}_{2} \mathrm{O}_{5}$, Wako Pure Chemical Industries, Ltd., Osaka, Japan) powders were hot-pressed to prepare the targets, Ag-TCP with and without Ta, at $1273 \mathrm{~K}$ and $20 \mathrm{MPa}$ for $7.2 \mathrm{ks}$ in an argon (Ar) atmosphere. An RF magnetron sputtering system (SSP 3000, SUGA Co., Ltd., Hokuto, Japan) was used for fabricating the films on CP Ti (JIS Gr. 2) substrates with a mirror-polished surface (average roughness: $\mathrm{Ra}<0.05 \mu \mathrm{m}$ ) and blasted Ti-6Al-4V substrates $(\mathrm{Ra}=4.6 \mu \mathrm{m}, 10 \mathrm{~mm} \times 10 \mathrm{~mm} \times 1 \mathrm{~mm})$. The two targets (Ag-TCP with and without Ta) with equivalent Ag concentrations of 10.0 mol\% were prepared. The sintered compacts are referred to as (Ta+Ag)-TCP and Ag-TCP, respectively. Table 1 shows the chemical composition of the targets used in this study.

Table 1. Notation and nominal measured compositions of targets used in this study (mol\%)

\begin{tabular}{llllll}
\hline Notation & & $\mathrm{Ca}$ & $\mathrm{P}$ & $\mathrm{Ag}$ & $\mathrm{Ta}$ \\
\hline \multirow{2}{*}{$\mathrm{Ag}-\mathrm{TCP}$} & Nominal & 54.0 & 36.0 & 10.0 & - \\
& Measured & 53.3 & 36.2 & 10.5 & - \\
\hline \multirow{2}{*}{ (Ta+Ag)-TCP } & Nominal & 48.6 & 32.4 & 10.0 & 9.0 \\
& Measured & 47.1 & 34.8 & 10.1 & 8.0 \\
\hline
\end{tabular}

The sputtering chamber was evacuated to a total pressure less than $9.9 \times 10^{-5} \mathrm{~Pa}$, and then highly pure Ar gas (99.9999 vol.\%) was introduced into the chamber. The Ar gas flow rate was $3.0 \times 10^{-7} \mathrm{~m}^{3} \cdot \mathrm{s}^{-1}$, and the total pressure $\left(P_{\text {tot }}\right)$ was maintained at $0.5 \mathrm{~Pa}$ in the chamber during sputtering. The targets were pre-sputtered for $1.2 \mathrm{ks}$ before

(C) The Authors, published by EDP Sciences. This is an open access article distributed under the terms of the Creative Commons Attribution License 4.0 (http://creativecommons.org/licenses/by/4.0/). 
the coating of the substrates. The substrate was deliberately not heated, and its distance from the targets was fixed at $70 \mathrm{~mm}$. The thickness of coating films was kept at 0.5 $\mu \mathrm{m}$ by changing the deposition time. Table 2 summarizes the deposition conditions.

The composition of the targets and the coating films were measured using an X-ray fluorescence spectrometer (XRF, ZSX Primus II, Rigaku Co., Tokyo, Japan). The phase of the coating film was identified using thin film XRD (RU-200B, Rigaku Co., Tokyo, Japan) with a low incident angle $\left(a=0.3^{\circ}\right)$. The cross section of the coating films was observed using a scanning transmission electron microscope (STEM, JEM-ARM200F, JEOL Ltd., Tokyo, Japan) at an accelerating voltage of $200 \mathrm{kV}$. The chemical state and distribution of elements in the coating films were evaluated using X-ray photoelectron spectroscopy (XPS, Axis-Ultra DLD, Shimadzu Co., Kyoto, Japan).

Table 2. Deposition conditions of coating films by radiofrequency magnetron sputtering

\begin{tabular}{ll}
\hline Deposition conditions & Deposition parameters \\
\hline Substrates & Mirror-polished CP Ti (JIS Gr. 2) \\
RF power $(\mathrm{P})$ & Blasted Ti-6Al-4V \\
Sputtering gas & $25-50 \mathrm{~W}$ \\
Total pressure $\left(\mathrm{P}_{\text {tot }}\right)$ & Argon \\
Target-substrate distance & $0.5 \mathrm{~Pa}$ \\
Thickness of coating films & $70 \mathrm{~mm}$ \\
\hline
\end{tabular}

Diluted NB (Sigma-Aldrich Inc., Darmstadt, Germany) and Tris-buffered solution (Tris, Wako Pure Chemical Industries, Ltd., Osaka, Japan) were used for the immersion tests. The NB solution was diluted 83-fold with purified water, based on the antibacterial test registered in ISO 27447 [17]. The initial pH of the Tris solution was maintained at 7.4 using $1 \mathrm{M}$ hydrochloric acid $(\mathrm{HCl}$, Kanto Chemical Co., Tokyo, Japan). The concentration of $\mathrm{Cl}$ ions in the Tris- $\mathrm{HCl}$ solution was approximately $30 \mathrm{mM}$. The coating films fabricated on blasted Ti-6Al-4V substrates using Ag-TCP and (Ta+Ag)-TCP targets were used for the immersion tests. A specimen was placed in a polypropylene bottle with 5 $\mathrm{mL}$ of solution, and then shaken in a water bath at $60 \mathrm{rpm}$ and $310 \mathrm{~K}$ for $86.4 \mathrm{ks}$. Afterwards, the substrate was picked up and rinsed with de-ionized water and dried in air. The surfaces of the coating films after immersion tests were analyzed using scanning electron microscopy with energy dispersive X-ray spectroscopy (SEM-EDX, VE-7800, Keyence Co., Osaka, Japan). $\mathrm{HNO}_{3}(5 \%, 0.3 \mathrm{~mL}$ ) was added to the immersed solutions to maintain the optimum pH for the following measurements. The concentration of released ions was measured using inductively coupled plasma-mass spectrometry (ICP-MS, Agilent 8800, Agilent Technologies Inc., Santa Clara, CA). The immersion tests were conducted in triplicates.

\section{Results and discussion}

3.1 Fabrication of the coating films

The coating films were fabricated by RF magnetron sputtering using Ag-TCP and (Ta+Ag)-TCP targets; hereafter, the coating films are referred as Ag-ACP and $(\mathrm{Ta}+\mathrm{Ag})$-ACP coating films, respectively. Table 3 indicates the chemical composition of the Ag-ACP and (Ta+Ag)-ACP coating films. The same concentration of Ag was achieved in the coating films with and without Ta. The XRD patterns (not shown) of both Ag-ACP and (Ta+Ag)-ACP coating films indicated that the phase of the coating films was amorphous calcium phosphate within a broad peak at around $30^{\circ}[5]$.

Table 3. Chemical composition of Ag-ACP and (Ta+Ag)-ACP coating films (mol\%)

\begin{tabular}{lccccc}
\hline Notation & $\mathrm{Ca}$ & $\mathrm{P}$ & $\mathrm{Ag}$ & $\mathrm{Ta}$ & $\mathrm{Ag} /(\mathrm{Ca}+\mathrm{Ag})$ atomic ratio \\
\hline $\mathrm{Ag}-\mathrm{ACP}$ & 59.9 & 31.1 & 9.0 & - & 0.13 \\
(Ta+Ag)-ACP & 50.3 & 23.4 & 11.7 & 14.7 & 0.19 \\
\hline
\end{tabular}

Cross-sectional TEM images of the obtained films are shown in Fig. 1. A uniform structure was observed inside both coating films. The coating films were dense and smooth, and no cracks or detachment from the substrates were observed. In addition, no diffraction rings were observed from the electron diffraction. These results indicated that the coating films were amorphous in phase, and both the added Ag and Ta were dissolved in ACP. Furthermore, the STEM mappings (not shown) show that all detected elements $(\mathrm{P}, \mathrm{Ca}, \mathrm{Ag}$, and $\mathrm{Ta}$ ) were homogeneously distributed in the coating films.
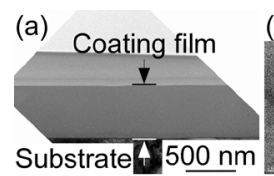

(d) Coating film

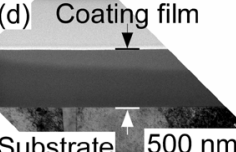

Substrate $500 \mathrm{~nm}$

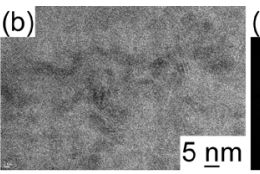

(e)
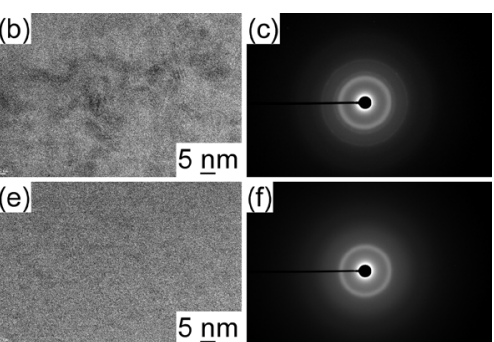

Fig. 1. TEM images and electron diffraction of (a-c) Ag-ACP and (d-f) (Ta+Ag)-ACP coating films.

The dissolution behavior of Ag in SBFs is known to be affected by the chemical state of $\mathrm{Ag}[18,19]$. XPS analysis determined that the chemical state of Ag in the coating films, dissolved in $\mathrm{ACP}$, was $\mathrm{Ag}^{+}$, which is consistent with the results observed by electron diffraction in Fig. 1. Keskar et al. synthesized Ag-loaded ACP powders by a spray pyrolysis method to obtain $\mathrm{ACP}$ with substituted $\mathrm{Ag}[20]$, and estimated an $\mathrm{Ag} /(\mathrm{Ca}+\mathrm{Ag})$ atomic ratio between 0.05 and 0.13 . In the present study, the measured $\mathrm{Ag} /(\mathrm{Ca}+\mathrm{Ag})$ atomic ratio in the ACP coating films was 0.13 or higher, reaching above 0.19 when there was co-doping with Ta (in Table 3). Therefore, the soluble Ag amounts in these coating films were higher than those reported by Keskar et al. These results suggest that the amorphous matrix can include considerable amounts of soluble Ag. In addition, the XPS also depicts that the chemical composition of each element was virtually constant along the depth direction (not shown). Such uniformity is consistent with results observed by STEM mappings. 
The concentrations of $\mathrm{Ca}$, P-related, $\mathrm{Ag}$, and Ta ions released from the coating films after immersion in diluted $\mathrm{NB}$ and Tris- $\mathrm{HCl}$ solutions for $86.4 \mathrm{ks}$ are presented in Fig. 2. Concentrations of $\mathrm{Ca}$ and P-related ions released from ( $\mathrm{Ta}+\mathrm{Ag})-\mathrm{ACP}$ coating film were smaller than those from Ag-ACP coating film in both solutions. The results of immersion test demonstrate that the dissolution of the Ag-ACP coating films could be effectively suppressed by the addition of Ta. Almost identical concentrations of Ag were detected in both coating films, meaning that both Ag-ACP and ( $\mathrm{Ta}+\mathrm{Ag}$ )-ACP coating films have potential antibacterial activity. On comparing the two solutions, the concentration of detected $\mathrm{Ag}$ ions in diluted $\mathrm{NB}$ was higher than that in Tris- $\mathrm{HCl}$ because of $\mathrm{AgCl}$ product formation due to the low solubility of AgCl. In both solutions, virtually the same concentration of Ta was released from the ( $\mathrm{Ta}+\mathrm{Ag})$-ACP coating films.
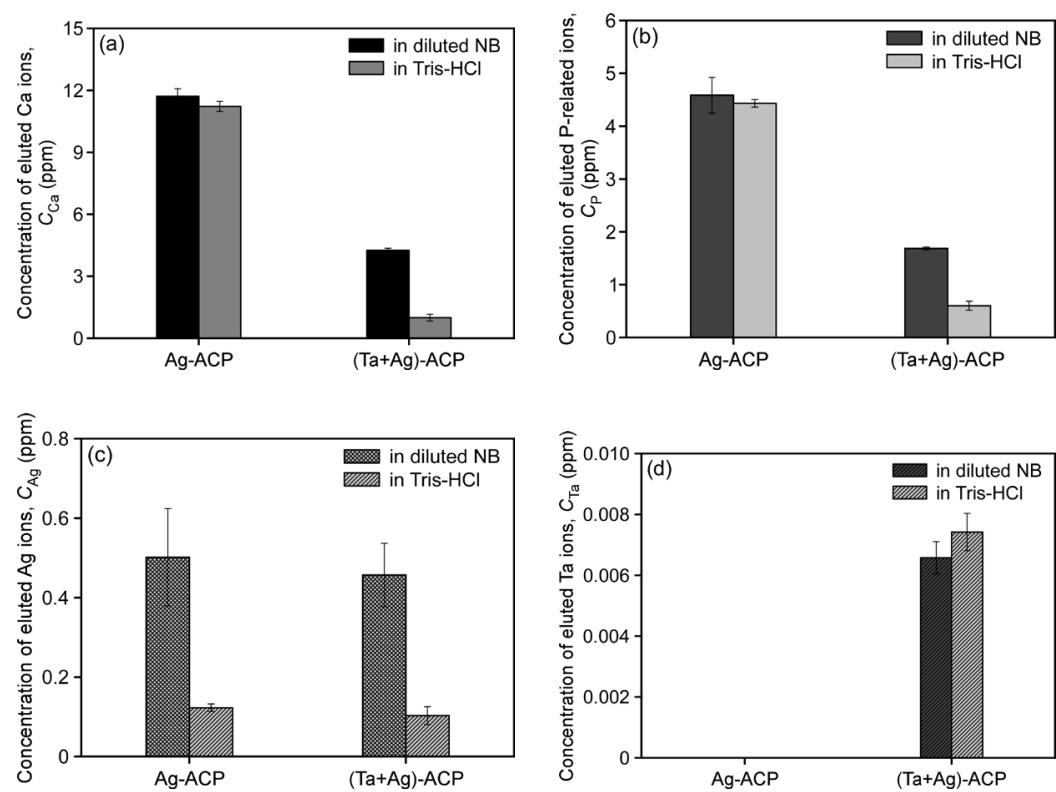

Fig. 2. Concentrations of (a) Ca, (b) P-related, (c) Ag, and (d) Ta ions in diluted nutrient broth (NB) and Tris-HCl solutions after immersing Ag-ACP and (Ta+Ag)-ACP coating films for $86.4 \mathrm{ks}$.

The EDX spectra of Ag-ACP and ( $\mathrm{Ta}+\mathrm{Ag}$ )-ACP coating films after the immersion test are shown in Fig. 3. In the Ag-ACP coating films, only Ag was detected as a substrate after the immersion test. In the ( $\mathrm{Ta}+\mathrm{Ag})$-ACP coating films, all elements ( $\mathrm{P}, \mathrm{Ca}, \mathrm{Ag}$, and $\mathrm{Ta}$ ) remained as substrates after the immersion test, indicating long-term antibacterial activity and bone formation. The antibacterial tests are on-going, and the results will be reported in the future.
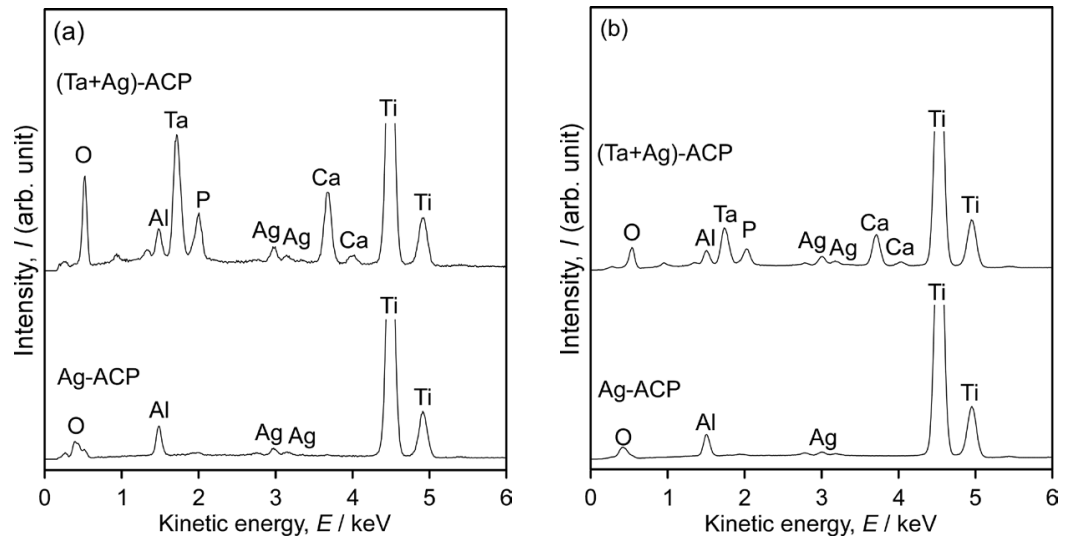

Fig. 3. Energy dispersive $\mathrm{X}$-ray (EDX) spectra of Ag-ACP and (Ta+Ag)-ACP coating films after immersing in (a) diluted nutrient broth (NB) and (b) TrisHCl solutions for $86.4 \mathrm{ks}$.

\section{Conclusion}

Ag- and Ta-containing ACP coating films were fabricated on Ti substrates by RF magnetron sputtering, and the following results were obtained:

1. Dense, smooth, and homogenous coating films with no cracks or detachment from the substrate were obtained.

2. Ag existed as an ion in the ACP coating films regardless of the addition of Ta.

3. The addition of Ta effectively suppressed the dissolution of ACP coating films. Ag was released from both the Ag-ACP and (Ta+Ag)-ACP coating films. 
The authors would like to thank Dr. Kobayashi of Tohoku University for his TEM analysis and Dr. Omura of Tohoku University for her XPS analysis. This study was financially supported by a Grant-in-Aid for Scientific Research from the Ministry of Education, Culture, Sports, Science and Technology (MEXT), Japan, under Contract Nos. 18H01718 and 17K06812. It was also supported by the Light Metal Educational Foundation, CRDAM-IMR, Tohoku University, and Creation of Life Innovation Materials for Interdisciplinary and International Researcher Development.

\section{References}

[1] M. Niinomi, Sci. Technol. Adv. Mater. 4 (2003) 445-454.

[2] T. Narushima, J. Japan Inst. Light Met. 55 (2005) 561-565.

[3] H. F. Morris, M. C. Manz, J. H. Tarolli, J. Oral Maxillofac. Surg. 55 (1997) 76-82.

[4] T. Thierer, J. P. Davliakos, J. D. Keith, J. J. Sanders, D. P. Tarnow, J. A. Rivers, J. Oral Implantol. 34 (2008) 39-46.

[5] T. Narushima, K. Ueda, T. Goto, H. Masumoto, T. Katsube, H. Kawamura, C. Ouchi, Y. Iguchi, Mater. Trans. 46 (2005) $2246-2252$.

[6] K. Ueda, T. Narushima, T. Goto, M. Taira, T. Katsube, Biomed. Mater. 2 (2007) S160-S166.

[7] K. Ueda, T. Narushima, T. Goto, T. Katsube, H. Nakagawa, H. Kawamura, M. Taira, Mater. Trans. 48 (2007) 307-312.

[8] K. Ueda, Y. Kawasaki, T. Narushima, T. Goto, J. Kurihara, H. Nakagawa, H. Kawamura, M. Taira, J. Biomech. Sci. Eng. 4 (2009) $392-403$.

[9] K. Ueda, T. Narushima, T. Goto, T. Katsube, H. Nakagawa, H. Kawamura, M. Taira, Mater. Sci. Forum. 631-632 (2009) 211-216.

[10] A. L. Boskey, J. Dent. Res. 76 (1997) 1433-1436.

[11] X. Yin, M. J. Stott, J. Chem. Phys. 118 (2003) 3717-3723.

[12] Y. Yang, C. M. Agrawal, K.-H. Kim, H. Martin, K. Schulz, J. D. Bumgardner, J. L. Ong, J. Oral Implantol. 29 (2003) 270-277.

[13] A. M. Alhalawani, M. R. Towler, Mater. Sci. Eng. C. 72 (2017) 202-211.

[14] N. J. Hickok, I. M. Shapiro, Adv. Drug Deliv. Rev. 64 (2012) 1165-1176.

[15] L. Crémet, S. Corvec, P. Bémer, L. Bret, C. Lebrun, B. Lesimple, A. F. Miegeville, A. Reynaud, D. Lepelletier, N. Caroff, J. Infect. 64 (2012) $169-175$.

[16] N. A. Trujillo, R. A. Oldinski, H. Ma, J. D. Bryers, J. D. Williams, K. C. Popat, Mater. Sci. Eng. C. 32 (2012) 2135-2144.

[17] I. Standard, ISO 27447, (2010).

[18] O. Gokcekaya, K. Ueda, T. Narushima, C. Ergun, Mater. Sci. Eng. C. 53 (2015) 111-119.

[19] O. Gokcekaya, K. Ueda, K. Ogasawara, H. Kanetaka, T. Narushima, Mater. Sci. Eng. C. 75 (2017) 926-933.

[20] M. Keskar, C. Sabatini, C. Cheng, M. T. Swihart, Nanoscale Adv. (2019) 1-9. 